\title{
Autophagy is Required for the Maintenance of Liver Progenitor Cell Functionality
}

\author{
Yiji Cheng ${ }^{a}$ Bei Wang ${ }^{b}$ Hong Zhou ${ }^{a}$ Shipeng Dang ${ }^{a}$ Min Jin ${ }^{a}$ Yufang Shi ${ }^{a}$ Li \\ $\mathrm{Hao}^{c}$ Zhenxi Yang ${ }^{\mathrm{d}}$ Yanyun Zhang ${ }^{\mathrm{a}}$ \\ aKey Laboratory of Stem Cell Biology, Institute of Health Sciences, Shanghai Institutes for Biological \\ Sciences, Chinese Academy of Sciences \& Shanghai Jiao Tong University School of Medicine, Shanghai, \\ ${ }^{b} X i n$ Hua Hospital Affiliated to Shanghai Jiao Tong University School of Medicine, Shanghai, 'Clinical \\ Laboratory, The First Affiliated Hospital of Anhui Medical University, Hefei, dDepartment of Surgery, \\ Shanghai Seventh People's Hospital, Shanghai, China
}

\section{Key Words}

Autophagy • Self-renewal • Hepatic differentiation • Cellular senescence

\begin{abstract}
Background: Liver progenitor cells (LPCs) are bipotent stem cells existing in the adult liver, which could be activated upon massive liver injury and contribute to liver regeneration. However, mechanisms of maintenance of LPC functionality remain poorly understood. Previous studies found that autophagy was required for the self-renewal and differentiation of several tissue stem cells. Methods: The study compared the level of autophagic activity in LPCs and differentiated hepatocytes. Then, autophagic activity was inhibited in LPCs by lentivirus-mediated autophagy-related gene 5 or Beclin 1 knockdown. Clonogenic assay, cell viability assays, hepatic differentiation assay, and senescence analysis were conducted to assess the role of autophagy in regulating self-renewal, hepatic differentiation and senescence of LPCs. Results: We observed high autophagic activity in LPCs compared with differentiated hepatocytes. We found that inhibition of autophagy impaired the self-renewal, proliferation, and hepatic differentiation capability of LPCs under normal cultural condition, but had little impact on cell viability. Interestingly, while wild-type LPCs remained rarely affected by the toxin, etoposide, inhibition of autophagy induced the senescent phenotype of LPCs. Overexpression of Beclin 1 in Beclin 1-knockdown LPCs restored the functionality of stem cells. Conclusion: Our findings indicate that autophagy may function as a critical regulator of LPC functionality under both physiological and pathological condition.
\end{abstract}




\section{Cellular Physiology Cell Physiol Biochem 2015;36:1163-1174 \begin{tabular}{l|l|l}
\hline DOI: 10.1159/000430287 & (C) 2015 S. Karger AG, Basel
\end{tabular} \begin{tabular}{l|l|} 
and Biochemistry Published online: June 25, 2015 & www.karger.com/cpb \\
\cline { 1 - 2 }
\end{tabular} \\ Cheng et al.: Autophagy Regulates Liver Progenitor Cells}

\section{Introduction}

Stem cells are characterized by their stemness: the capability of self-renewal and multipotential differentiation [1]. Liver progenitor cells (LPCs) are bipotent cells which can self-renew and differentiate into hepatocytes and biliary epithelial cells under specific condition [2]. In rodents, LPCs are also called oval cells because of the "oval like" shape and large nuclear-to-cytoplasmic ratio [3]. Upon massive liver damage, LPCs can be activated, proliferate and finally differentiate into functional mature cells to promote liver regeneration. In mice, LPCs are often observed in mice fed with a choline-deficient ethioninesupplemented (CDE) diet [4] or a 3,5-diethoxycarbonyl-1,4-dihydrocollidine diet [2]. In humans, LPC proliferation is often observed in a variety of liver diseases, including fatty liver diseases [5], chronic viral hepatitis [6], and cirrhosis [7]. Due to their bipotential property, LPCs are considered as potential target for liver cell transplantation and therapeutic liver repopulation [8]. Thus, mechanisms of LPC regulation under physiological and pathological condition are needed to be explored. However, the regulation of LPC functionality is not fully understood.

Autophagy is the conserved catabolic mechanism involving cell degradation of unnecessary or dysfunctional cellular components by lysosomes [9]. There are three types of autophagy, including macroautophagy, microautophagy and chaperone-mediated autophagy [10]. Among them, macroautophagy is the most extensively studied pathway. Macroautophagy (simply referred to as autophagy hereafter) regulates a variety of physiological and pathological events, including tumor suppression, innate and adaptive immunity, lifespan extension and cell death [11]. Although autophagy in somatic cells has been widely explored, the role of autophagy in the maintenance and function of stem cells is only beginning to be revealed as a result of recent studies. Our previous study on mesenchymal stem cells (MSCs) has found that autophagy was induced in MSCs by inflammatory cytokines, and this inflammatory microenvironment-induced autophagy inhibits the immunosuppressive function of MSCs, indicating autophagy as a novel target to improve MSC-based immunotherapy [12]. Moreover, others have found that autophagy in MSCs was important for the protection of stemness from irradiation injury [13]. In addition, in adult hematopoietic stem cells, autophagy was also important for the stemness maintenance and differentiation [14]. However, the role of autophagy in LPC regulation is still unknown and needs to be explored.

In this study, we investigated the role of autophagy in regulating LPC functionality, including self-renewal, proliferation, differentiation and cellular senescence. Interestingly, we found that inhibition of autophagy in LPCs by knockdown of the essential autophagy genes autophagy-related gene 5 (Atg5) or Beclin 1 (Becn1) impaired the stemness of LPCs, and promoted the senescence of LPCs induced by etoposide.

\section{Materials and Methods}

\section{Animals}

C57BL/6 mice of 8-12 weeks old were used for isolation of murine LPCs. Mice were fed with a CDE (TROPHIC, Nantong, Jiangsu, China) diet to induce LPC activation as previously reported [4] and subject to LPC isolation. All these experiments with animals were performed in accordance with the relevant guidelines and regulations of Shanghai Jiao Tong University School of Medicine. All the experiments with animals were approved by the Animal Welfare \& Ethics Committee of Shanghai Jiao Tong University School of Medicine.

\section{Isolation of hepatocytes}

Hepatocytes were isolated from naïve mice using the two-step perfusion protocol as previously described [15]. After the mice were anesthetized, mouse livers were first perfused with perfusion buffer, 


\section{Cellular Physiology Cell Physiol Biochem 2015;36:1163-1174 \begin{tabular}{l|l|l}
\cline { 2 - 2 } DOI: 10.1159/000430287 & C 2015 S. Karger AG, Basel
\end{tabular} \begin{tabular}{l|l} 
and Biochemistry Published online: June 25, 2015 & www.karger.com/cpb
\end{tabular} \\ Cheng et al.: Autophagy Regulates Liver Progenitor Cells}

and then with buffer containing collagenase D (Roche, Basel, Switzerland). Then, by shaking the liver in the collagenase buffer, cells were released from the liver. Liver nonparenchymal cells were removed from the hepatocytes by several episodes of low-speed centrifugation ( $40 \times \mathrm{g}$ for $3 \mathrm{~min}$ ). Those freshly isolated hepatocytes can be harvested for further analysis.

Isolation, Culture, and Clone of LPC Line

Primary murine LPCs were isolated from mice with CDE diet as previously described [16]. Briefly, after 3 weeks on the CDE diet, liver cells were then harvested by a modification of a multi-step digestion protocol. Liver nonparenchymal cells were separated from the hepatocytes by several episodes of lowspeed centrifugation (40×g for $3 \mathrm{~min}$ ) and were then collected by centrifugation (1500 rpm for $5 \mathrm{~min}$ ). LPCs were enriched by centrifugation of nonparenchymal cells through a discontinuous gradient of $20 \%$ and 50\% Percoll ${ }^{\mathrm{TM}}$ (Amersham Biosciences, Pittsburgh, PA, USA). Enriched LPCs were then incubated with APC-conjugated anti-EpCAM and FITC-conjugated anti-CD45 (eBioscience, San Diego, CA, USA). Then, $\mathrm{EpCAM}^{+} \mathrm{CD} 45$ cells were isolated by fluorescence-activated cell sorting (FACS) and plated in type I collagencoated dishes (BD Biosciences, San Jose, CA, USA). The cells were maintained in culture medium: DMEM/ F12 (Invitrogen, Carlsbad, CA, USA) with 10\% fetal bovine serum (FBS, Invitrogen), $10^{-7} \mathrm{M}$ dexamethasone, $1 \times$ ITS-X (insulin, transferrin, selenium X, Invitrogen), $10 \mathrm{ng} / \mathrm{ml}$ hepatocyte growth factor (HGF), $20 \mathrm{ng} / \mathrm{ml}$ epidermal growth factor (EGF) and $20 \mathrm{ng} / \mathrm{ml}$ fibroblast growth factor (FGF) (cytokines all from PeproTech, Rocky Hill, NJ, USA). The culture medium was half changed every 2 days. The purified epithelial populations were named LPCs and passaged every 3 days.

\section{Induction of hepatic differentiation}

To induce hepatic differentiation, LPCs were grown to confluence, washed with PBS twice, and cultured in medium supplemented with 20\% Matrigel, $40 \mathrm{ng} / \mathrm{mL}$ oncostatin M (R\&D Systems, Minneapolis, MN, USA), $25 \mathrm{ng} / \mathrm{mL} \mathrm{HGF}, 25 \mathrm{ng} / \mathrm{mL}$ EGF, and $10^{-7} \mathrm{M}$ dexamethasone for 7 days [17]. Then, cells were harvested for western blot analysis or subjected to PAS staining. Cells grown on chamber slides were fixed with $4 \%$ paraformaldehyde (PFA) for $30 \mathrm{~min}$ and washed with PBS twice. Next, slides were incubated in periodic acid solution for $5 \mathrm{~min}$ and rinsed with several changes of distilled water. Slides were incubated in Schiff's reagent (Sigma, St. Louis, MO, USA) for $15 \mathrm{~min}$, washed in running tap water for $5 \mathrm{~min}$, dehydrated, and mounted with xylene-based mounting medium.

\section{IF staining}

LPCs with differential treatment were fixed with 4\% PFA, washed with PBS and incubated with $0.2 \%$ Triton X-100. Then, the cells were incubated with anti-LC3 antibody (Cell Signaling Technology, Danvers, MA, USA) in 1\% bovine serum albumin. Secondary antibody was Cy3-conjugated antibody (Invitrogen). For counterstaining of the nucleus, cells were incubated with DAPI. Finally, cells were mounted and observed under a confocal microscope, LSM510 (Carl Zeiss, Oberkochen, Germany).

\section{Lentiviral vector construction}

Lentiviral shRNA vectors (GenePharma, Shanghai, China) targeting the essential autophagy genes Atg5/Becn1 were utilized for stable knockdown in LPCs. Procedures were conducted according to the manufacturer's protocol. shRNA sequences were as follows: shAtg5, 5'-gat ccG CGG TTG AGG CTC ACT TTA TGT TCA AGA GAC ATA AAG TGA GCC TCA ACC GCT TTT TTg-3'; shBecn1, 5'-gat ccG GAG AAA GGC AAG ATT GAA GAT TCA AGA GAT CTT CAA TCT TGC CTT TCT CCT TTT TTg-3'; shNC, 5'-gat ccA CTA CCG TTG TTA TAG GTG TTC AAG AGA CAC CTA TAA CAA CGG TAG TTT TTT Tg-3' [12].

High titer lentiviral stocks were produced and used at a multiplicity of infection of 50 to infect LPCs, and the efficiency of infection exceeded 95\%. LPCs were infected with lentivirus expressing shNC or shRNA inhibiting Atg5 or Becn1.

\section{Cell proliferation assay}

LPCs with differential treatment were seeded in 96-well plate at 5000 cells per well. CCK8 assay (Dojindo Laboratories, Kumamoto, Japan) was used to measure the viable proliferating cells at 0, 24, 48, 72 and 96 hours after plating. The absorbance at $450 \mathrm{~nm}$ was measured using a thermo Spectrophotometer. 


\section{Cellular Physiology Cell Physiol Biochem 2015;36:1163-1174 \begin{tabular}{l|l|l}
\hline DOI: 10.1159/000430287 & C 2015 S. Karger AG, Basel
\end{tabular} \begin{tabular}{l|l} 
and Biochemistry Published online: June 25, 2015 & www.karger.com/cpb
\end{tabular} \\ Cheng et al.: Autophagy Regulates Liver Progenitor Cells}

\section{Cell viability assays}

Cultured LPCs were harvested and subjected to trypan blue exclusion assay or PI staining to assess cell viability. For trypan blue exclusion assay, cells were mixed with an equal volume of trypan blue solution (Beyotime, Nantong, Jiangsu, China) and counted under microscope. The number and percentage of dead cells were quantified. For PI staining, cells were stained with PI staining solution (eBioscience, San Diego, CA, USA) according to the manufacturer's instruction. Stained cells were analyzed using FACSCaliber (BD Biosciences, San Jose, CA, USA).

PCR

Total RNA was extracted from LPCs with differential treatment at indicated times and was subsequently reverse-transcribed using AMV Reverse Transcription System (Takara, Shiga, Japan). Real-time PCR was performed using SYBR Green PCR mix (Roche, Basel, Switzerland) on an ABI Prism 7900HT (Applied Biosystems, Foster city, CA, USA). Thermocycler conditions included 2-minute incubation at $50^{\circ} \mathrm{C}$, then $95^{\circ} \mathrm{C}$ for 10 minutes; this was followed by a 2-step PCR program, as follows: $95^{\circ} \mathrm{C}$ for 15 seconds and $60^{\circ} \mathrm{C}$ for 60 seconds for 40 cycles, as previously described [18]. $\beta$-actin was used as an internal control to normalize for differences in the amount of total RNA in each sample. The primer sequences were as follows (in the 5 ' to 3 ' orientation): $\beta$-actin forward, TGT CCA CCT TCC AGC AGA TGT; $\beta$-actin reverse, AGC TCA GTA ACA GTC CGC CTA GA; p53 forward, GCC GAC CTA TCC TTA CCA TCA; p53 reverse, GGC AGG CAC AAA CAC GAA C; p21 forward, CCT GGT TCC TTG CCA CTT CTT; p21 reverse, CTG TTC TAG GCT GTG ACT GCT TC; p16 forward, CGT TCA CGT AGC AGC TCT TC; 16 reverse, GCA CGA TGT CTT GAT GTC CC; albumin forward, TGG GTA ACC TTT CTC CTC CTC C; albumin reverse, CAC TCT TGT GTG CTT CTC GGC; G-6-Pase forward, CAT CAA TCT CCT CTG GGT GGC; G-6-Pase reverse, CGT TGC TGT AGT AGT CGG TGT CC; CK-19 forward, ACC CTC CCG AGA TTA CAA CCA C; CK-19 reverse, CAA GGC GTG TTC TGT CTC AAA C; CK-7 forward, AGG AGA TCA ACC GAC GCA C; CK-7 reverse, GTC TCG TGA AGG GTC TTG AGG.

\section{Western Blot}

Cells were harvested and lysed with RIPA containing protease and phosphatase inhibitors (Roche) and proteins were separated by $12 \%$ - 14\% SDS-PAGE after denaturation. Immunoblot analysis was performed by initial transfer of proteins onto polyvinylidenefluoride membranes and followed by a blocking step with $5 \%$ nonfat dried milk plus $0.1 \%$ Tween 20 and exposed to primary antibodies against LC3-I/II, Atg5, Becn1, p62, PARP and GAPDH (all from Cell Signaling Technology). The blots were then incubated with a secondary antibody conjugated with Horse Radish Peroxidase (Cell Signaling Technology) and the immunoreactivity was detected by chemiluminescence (Merck Millipore, Billerica, MA, USA). Immunoblot bands were quantified by densitometry using the software ImageJ. Densities were normalized to control treatment and relative folds were normalized to GAPDH.

\section{Senescence analysis}

Senescence of LPCs was compared by histochemical staining for expression of SA- $\beta$-Gal activity, as previously described [19]. LPCs with differential treatment were gently rinsed with PBS, and fixed with $0.2 \%$ gluteraldehyde and $2 \%$ formaldehyde. The stain solution [ $\beta$-Gal Staining kit (Cell Signaling Technology)], adjusted to $\mathrm{pH} 6.0$, was added to each culture plate and incubated for 16 hours at $37^{\circ} \mathrm{C}$. The plates were then removed from the incubator and rinsed with PBS after the staining substrate was removed. Plates were then coverslipped for viewing and photography.

\section{Clonogenic assay}

LPCs were plated at 250 cells per 100-mm dish and cultured in the DMEM/F12 containing 10\% FBS, $10^{-7} \mathrm{M}$ dexamethasone, $1 \times$ ITS-X, $10 \mathrm{ng} / \mathrm{ml} \mathrm{HGF,} 20 \mathrm{ng} / \mathrm{ml} \mathrm{EGF,} 20 \mathrm{ng} / \mathrm{ml} \mathrm{FGF}$, and $20 \mu \mathrm{M}$ Y27632 (Sigma). The cells were cultured for 9 days and then the numbers of colonies were counted. To visualize clonogenic expansion, cell cultures were fixed in 10\% formalin, stained with crystal violet/methanol (Beyotime, Nantong, Jiangsu, China), and imaged using conventional scanning and imaging tools.

\section{Transfection}

The myc-tagged full-length Beclin 1 plasmid or control vector were kindly gifted from Dr Congfeng Xu at Shanghai Jiao Tong University School of Medicine, Shanghai, China [20]. LPCs were transfected by using Lipofectamine 2000 (Invitrogen, Carlsbad, CA, USA).

\section{KARGER}




\section{Cellular Physiology Cell Physiol Biochem 2015;36:1163-1174 \begin{tabular}{l|l|l}
\hline DOI: 10.1159/000430287 & (C) 2015 S. Karger AG, Basel
\end{tabular} www.karger.com/cpb

\section{Statistical analysis}

Differences were evaluated using SPSS (Version 16.0, Chicago, IL, USA). Significant differences were evaluated using an independent-samples $t$ test, except that multiple treatment groups were compared within individual experiments by ANOVA. All values were presented as mean \pm S.E.M. Values of $P$ less than 0.05 were considered significant.

\section{Results}

\section{Autophagy in wild-type LPCs and inhibition of autophagy}

Microtubule-associated protein light chain 3 (LC3) expression is the most commonly used indicator for autophagosome formation [10]. To investigate the level of autophagic activity in LPCs, we have analyzed the expression of autophagic markers, LC3-I/ II and p62, in freshly isolated LPCs and hepatocytes. LPCs and hepatocytes were isolated as described in Materials and Methods, and then were cultured in DMEM/F12 medium with/without the lysosomal inhibitor chloroquine $(3 \mu \mathrm{M})$ for 3 hours. After the treatment, cells were harvested and proteins were collected for western blot analysis. As shown in Fig. 1A, expression of LC3II in freshly isolated LPCs was higher than that in freshly isolated hepatocytes, both in the presence and absence of chloroquine. Meanwhile, expression of p62 in freshly isolated LPCs was lower than that in freshly isolated hepatocytes, both in the presence and absence of

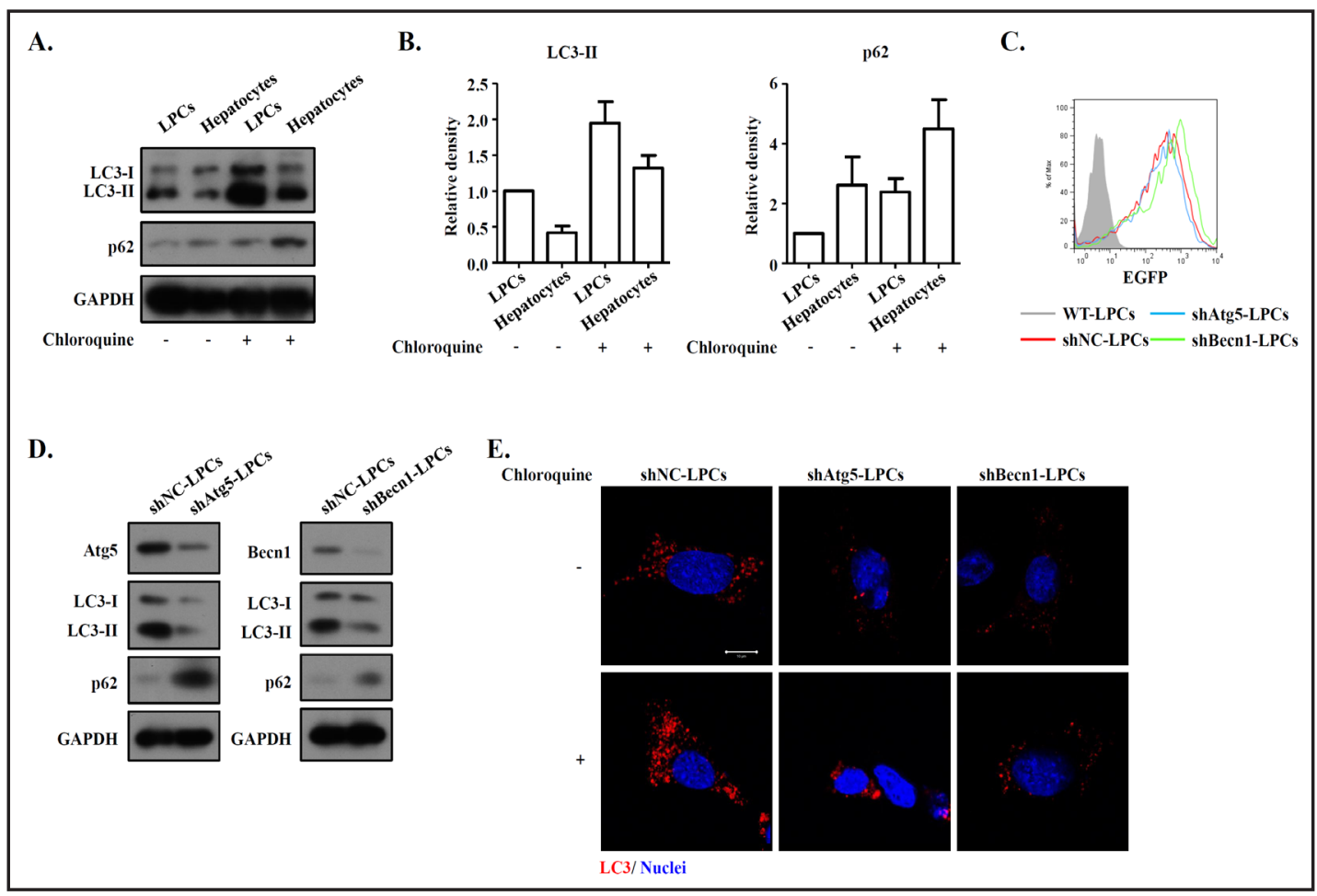

Fig. 1. Level of autophagy in wild-type and Atg5/Becn1-knockdown LPCs. (A) LPCs and hepatocytes were isolated as described in Materials and Methods, and then were cultured in DMEM/F12 medium with/without the lysosomal inhibitor chloroquine $(3 \mu \mathrm{M})$ for 3 hours. After the treatment, cells were harvested and proteins were collected for western blot analysis. Western blot analysis of LC3-I/II and p62 expression in LPCs and hepatocytes. Representative data from 3 separate experiments are shown. (B) Densities of LC3II or p62 were normalized to control group and relative fold changes of LC3-II or p62 were normalized to GAPDH. (C) The EGFP-positivity of cells transduced with indicated viruses. (D) Western blot analysis of Atg5, Becn1, LC3-I/II and p62 expression in cultured shNC/shAtg5/shBecn1-LPCs. (E) shNC/shAtg5/shBecn1-LPCs treated with/without the lysosomal inhibitor chloroquine were labeled with anti-LC3 primary antibody and Cy3-conjugated secondary antibody. IF staining was analyzed by confocal microscopy. Scale bar $=10 \mu \mathrm{m}$. Results are representative of three independent experiments with similar results. 


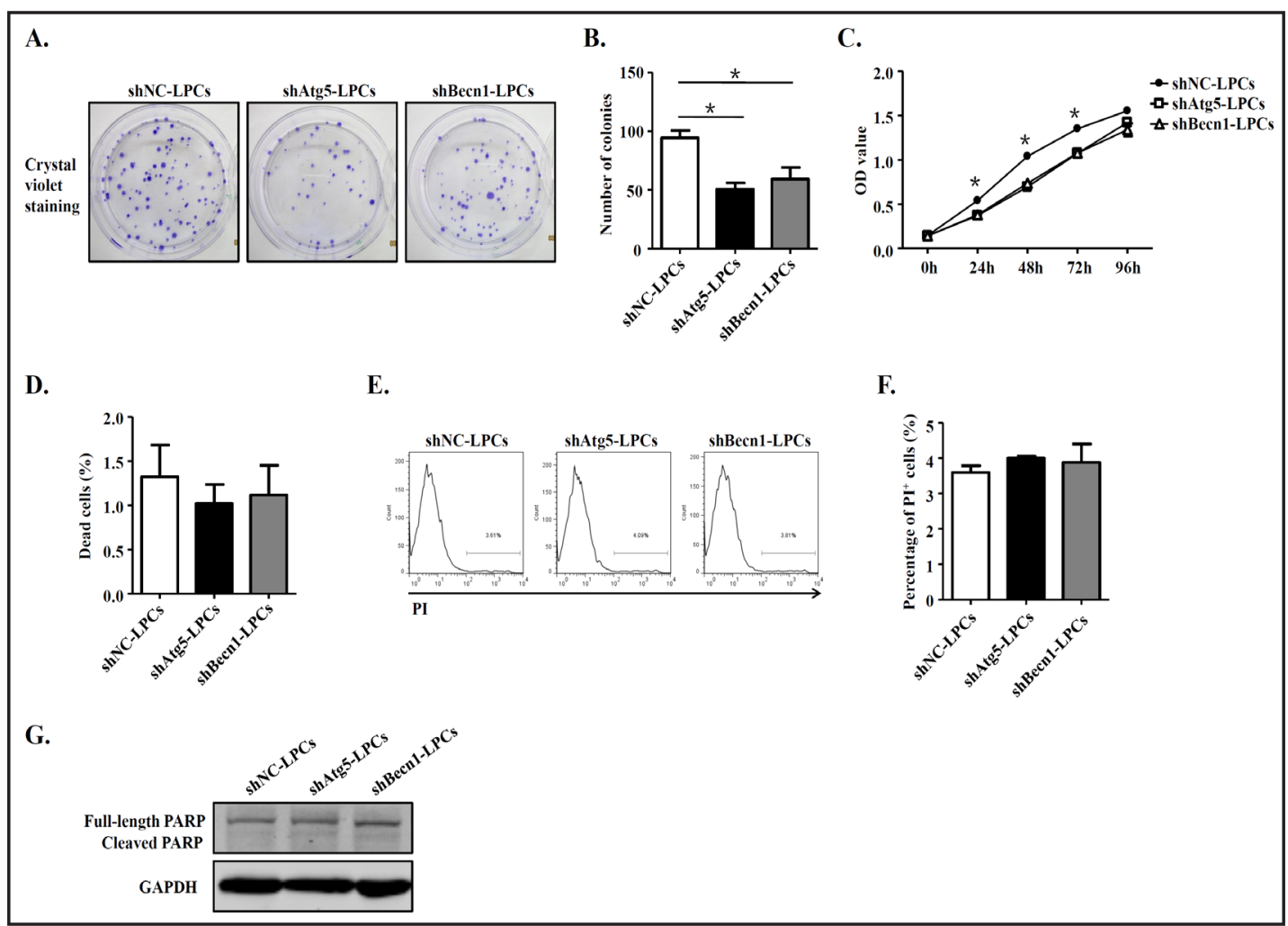

Fig. 2. Inhibition of autophagy limited the clonogenic and proliferative capability of LPCs. (A) Clonogenic colony-forming assay of cultured shNC/shAtg5/shBecn1-LPCs. (B) Colony number of data depicted in A was quantified. (C) Proliferation of shNC/shAtg5/shBecn1-LPCs in vitro was analyzed by CCK-8 assay. ${ }^{*} P<0.05$. (D) Cell viability was analyzed by trypan blue exclusion assay. The percentage of dead cells was quantified. (E) Cell viability was analyzed by PI staining using flow cytometry. (F) The percentage of $\mathrm{PI}^{+}$cells depicted in E was quantified. (G) Expression of full-length and cleaved PARP in shNC/shAtg5/shBecn1-LPCs was analyzed by western blot. Results are representative of three independent experiments with similar results.

chloroquine. Quantification of LC3-II and p62 also confirmed the results (Fig. 1B), indicating the high autophagic activity in LPCs compared to hepatocytes.

To investigate the function of autophagy in LPCs, we used lentivirus-mediated Atg5/ Becn1-knockdown. As Atg5 and Becn1 are essential autophagy genes, knockdown of Atg5/ Becn1 in LPCs would inhibit autophagic activity. LPCs were then infected with shNC/ shAtg5/shBecn 1 viruses (shNC/shAtg5/shBecn1-LPCs) and allowed to propagate for 3 days. Flow cytometric analysis revealed that the majority (more than 90\%) of infected LPCs were positive for enhanced green fluorescent protein (EGFP), a marker of lentiviral integration (Fig. 1C). Western blot confirmed the successful knockdown of Atg5/Becn1 and inhibition of autophagy in shAtg5/shBecn1-LPCs compared with shNC-LPCs, as evidenced by the decreased expression of LC3-II and increased expression of p62 (Fig. 1D). In addition, we have examined the LC3 puncta in shNC/shAtg5/shBecn1-LPCs treated with or without the lysosomal inhibitor, chloroquine. As shown by immunofluorescence staining, the number of LC3 puncta in shNC-LPCs was higher than those in shAtg5/shBecn1-LPCs, both in the presence and absence of lysosomal inhibitor chloroquine (Fig. 1E).

\section{Impaired self-renewal and proliferative capability of LPCs following inhibition of autophagy}

Stem cells and/or progenitor cells are characterized by their ability to self-renew. To investigate the effect of autophagy on the self-renewal of LPCs, shNC/shAtg5/shBecn1-LPCs were used in the following experiments. Efficiency of LPC self-renewal was assessed by the 


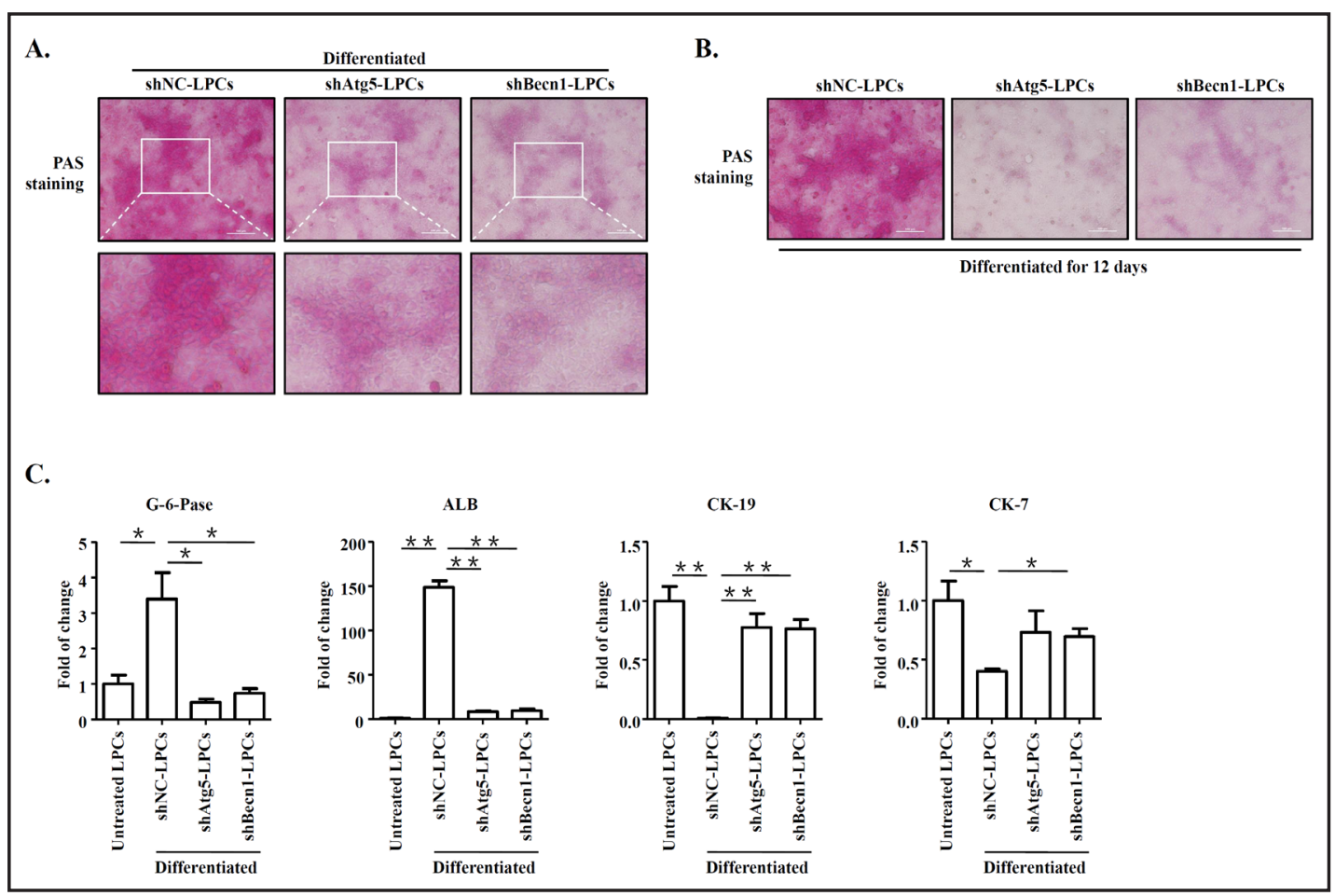

Fig. 3. Inhibition of autophagy decreased the hepatic differentiation of LPCs. (A) shNC/shAtg5/shBecn1-LPCs were cultured in hepatic differentiation media for 7 days and subjected to PAS staining. Scale bar $=100$ $\mu \mathrm{m}$. (B) shNC/shAtg5/shBecn1-LPCs were cultured in hepatic differentiation media for 12 days and subjected to PAS staining. Scale bar $=100 \mu \mathrm{m}$. (C) Expression of G-6-Pase, ALB, CK-19 and CK-7 was analyzed by real-time PCR. RNA was prepared from untreated shNC-LPCs and differentiated shNC/shAtg5/shBecn1-LPCs. Expression of G-6-Pase, ALB, CK-19 and CK-7 mRNA was normalized to $\beta$-actin. Results are representative of three independent experiments with similar results. ${ }^{*} P<0.05,{ }^{* *} P<0.01$.

rate of colony formation in colony-forming unit (CFU) assay. As indicated in Fig. 2A \& B, CFU numbers in shAtg5/shBecn1-LPCs were significantly decreased compared with that in shNCLPCs. In addition, in vitro proliferation assay demonstrated that the level of proliferation in shAtg5/shBecn1-LPCs was significantly lower than that in shNC-LPCs at 24, 48 and 72 hours after plating (Fig. 2C).

Meanwhile, we have also performed cell viability assays to test whether inhibition of autophagy would affect cell viability. shNC/shAtg5/shBecn1-LPCs were harvested and subjected to trypan blue exclusion assay or Propidium Iodide (PI) staining, and cell viability was observed under microscope or detected by flow cytometry analysis. The reason we didn't stain the cells with both PI and Annexin V-FITC to test apoptosis was that shNC/shAtg5/ shBecn1-LPCs were GFP positive due to the lentiviral system. As indicated by trypan blue exclusion assay (Fig. 2D) and flow cytometry analysis (Fig. 2E,F), shNC/shAtg5/shBecn1LPCs displayed similar viabilities. In addition, western blot analysis demonstrated that shNC/shAtg5/shBecn1-LPCs expressed similar levels of full-length and cleaved poly (ADPribose) polymerase (PARP), which is a marker for apoptosis (Fig. 2G).

Together, these results suggested that inhibition of autophagy impaired the self-renewal and proliferative capability of LPCs, but had little impact on cell viability.

Inhibition of autophagy decreased the hepatic differentiation of LPCS

LPCs could differentiate into hepatocytes under specific condition. To evaluate the involvement of autophagy in the hepatic differentiation of LPCs, shNC/shAtg5/shBecn1LPCs were subjected to hepatic differentiation media. Periodic acid-Schiff (PAS) staining demonstrated that glycogen deposition was detected in shNC-LPCs, indicating that shNC- 


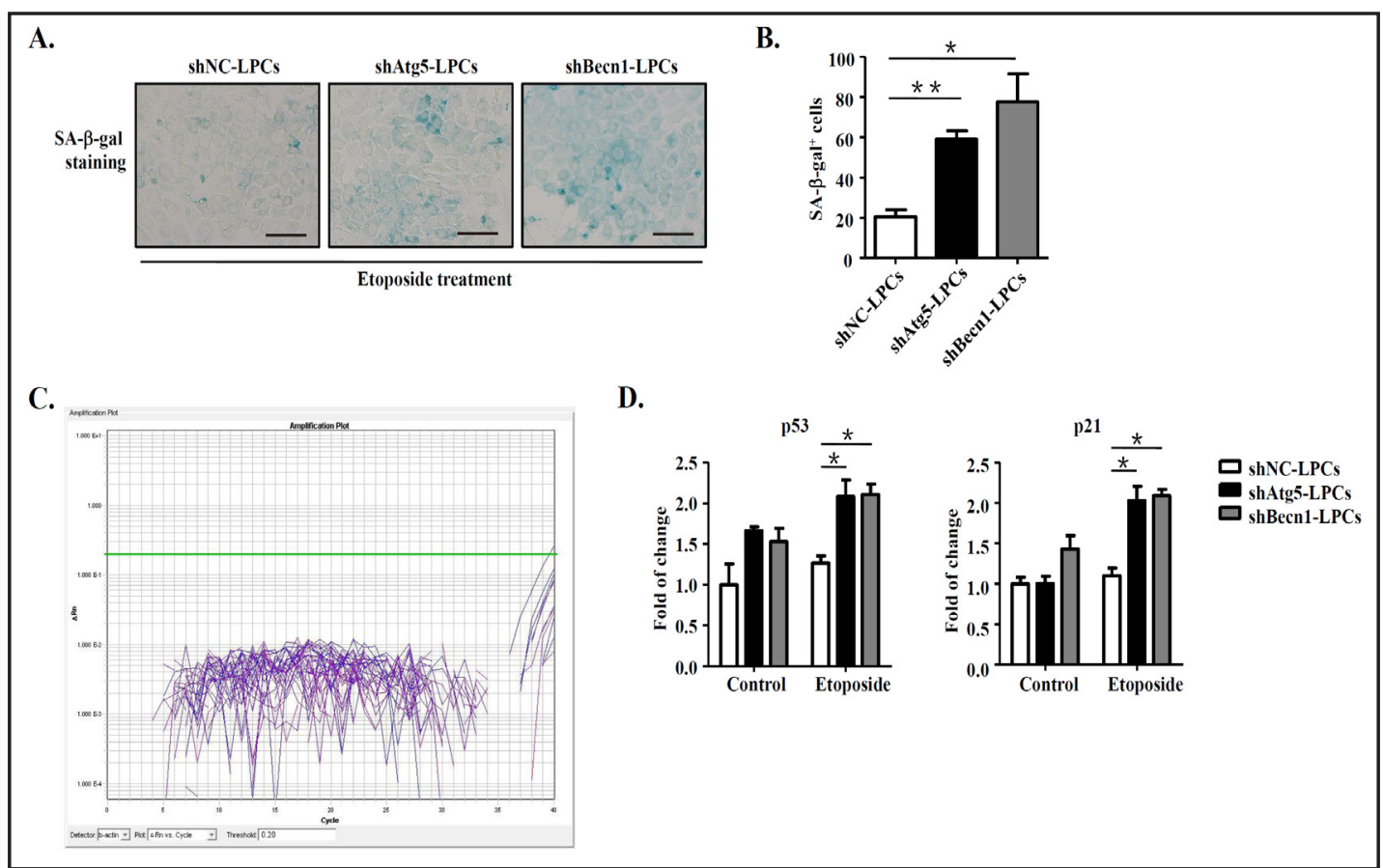

Fig. 4. Inhibition of autophagy promoted the senescence of LPCs in response to etoposide stimulation. Cultured shNC/shAtg5/shBecn1-LPCs were treated with $25 \mu \mathrm{M}$ etoposide for 24 hours. (A) SA- $\beta$-gal staining of shNC/shAtg5/shBecn1-LPCs treated with etoposide. Scale bar $=100 \mu \mathrm{m}$. (B) Number of SA- $\beta$-gal ${ }^{+} L P C s$ depicted in A was quantified. (C) Expression of p16 in LPCs was analyzed by real-time PCR. The amplification plot of p16 was demonstrated using the software SDS 2.3 (Applied Biosystems, Grand Island, NY, USA). (D) p53 and p21 mRNA expression was measured by real-time PCR. $* P<0.05, * * P<0.01$. Results are representative of three independent experiments with similar results.

LPCs could differentiate into hepatocytes upon induction (Fig. 3A). However, shAtg5/ shBecn1-LPCs exhibited decreased potential of differentiating into hepatocytes (Fig. 3A). Interestingly, when the hepatic differentiation period was extended to 12 days, shAtg5/ shBecn1-LPCs still could not fully differentiate into hepatocytes (Fig. 3B).

In addition to PAS staining, we have also analyzed the expression of hepatocyte markers glucose 6-phosphatase (G-6-Pase) and albumin (ALB), and cholangiocyte markers cytokeratin-19 (CK-19) and CK-7, by real-time PCR. Real-time PCR analysis demonstrated that shNC-LPCs upon hepatic differentiation induction expressed higher levels of G-6-Pase and ALB, compared with shAtg5/shBecn1-LPCs upon hepatic differentiation (Fig. 3C). Meanwhile, the expression of CK-19 and CK-7 was decreased in shNC-LPCs upon hepatic differentiation (Fig. 3C). However, the expression of these two cholangiocyte markers was not significantly changed in shAtg5/shBecn1-LPCs upon hepatic differentiation (Fig. 3C). These results further confirmed our conclusion that inhibition of autophagy inhibited the hepatic differentiation of LPCs.

\section{Inhibition of autophagy promotes senescence of LPCS}

Etoposide is a DNA damaging drug which could cause senescent morphology in various cells [21]. We treated shNC/shAtg5/shBecn1-LPCs with $25 \mu \mathrm{M}$ etoposide. After 24 hours, etoposide caused senescent morphology in shNC/shAtg5/shBecn1-LPCs as evidenced by senescence associated $\beta$-galactosidase (SA- $\beta$-Gal) staining (Fig. 4A). However, shAtg5/ shBecn1-LPCs were rather sensitive to etoposide-induced damage compared with shNCLPCs, as evidenced by the large number of SA- $\beta-\mathrm{Gal}^{+}$cells in shAtg5/shBecn1-LPCs than that in shNC-LPCs (Fig. 4A,B), suggesting inhibition of autophagy rendered LPCs susceptible to stress-induced senescence. 
Fig. 5. Overexpression of Beclin 1 in shBecn1-LPCs restored the functionality of stem cells. Cultured shBecn1-LPCs were transiently transfected with control vector or expression vector for Beclin 1. (A) Expression of Beclin 1, LC3-I/II was analyzed by western blot. (B) shBecn1-LPCs transiently transfected with control vector or expression vector for Beclin 1 were cultured in hepatic differentiation media for 7 days and subjected to PAS staining. Scale bar $=100$ $\mu \mathrm{m}$. (C) shBecn1-LPCs transiently transfected with control vector or expression vector for Beclin 1 were treated with $25 \mu \mathrm{M}$ etoposide for 24 hours. SA- $\beta$-gal staining of shBecn1-LPCs with etoposide was displayed. Scale bar $=100 \mu \mathrm{m}$. (D) Number of SA- $\beta$-gal ${ }^{+}$senescent cells depicted in $\mathrm{C}$ was quantified. ** $P<0.01$

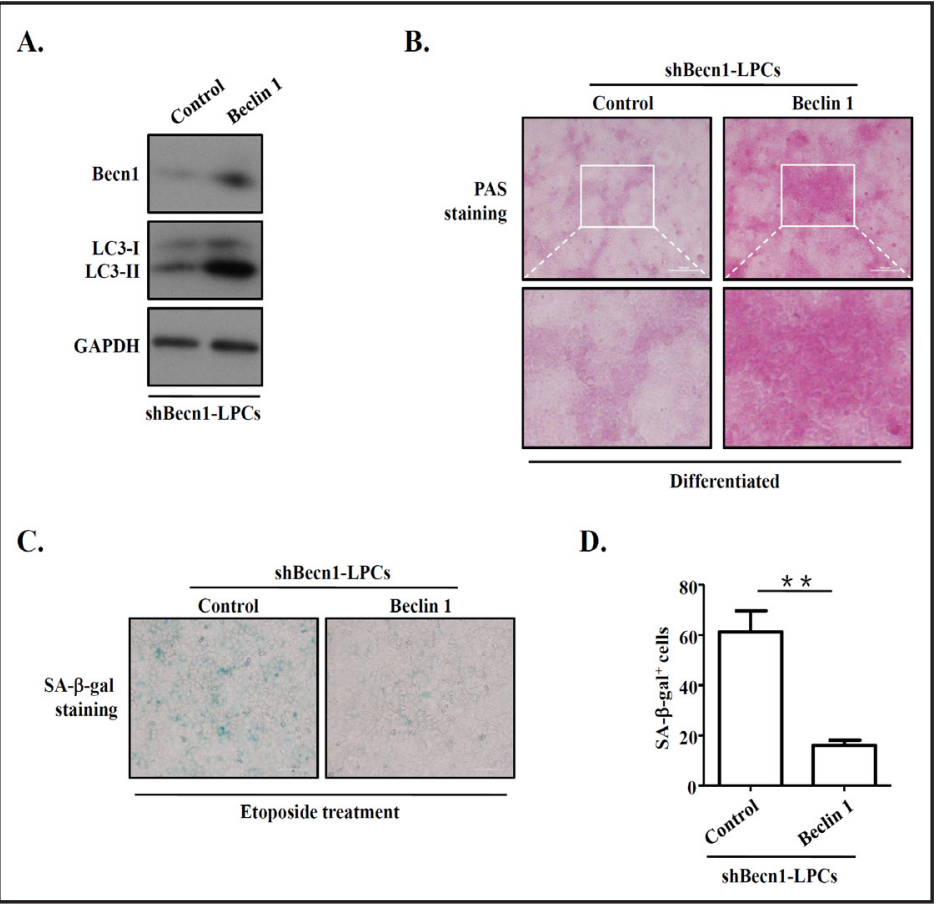

Then, we investigated the mechanism by which autophagy regulated the senescence of LPCs induced by etoposide. It is known that p16/pRB and p53/p21 are two major senescence-triggering pathways in response to stresses [22]. Therefore, we detected the expressions of p53, p21 and p16 in shNC/shAtg5/shBecn1-LPCs treated with or without etoposide using real-time PCR analysis. While mRNA expression of p16 was not detected in LPCs (Fig. 4C), mRNA expression of p53 and p21 were significantly increased in shAtg5/ shBecn1-LPCs treated with etoposide compared to shNC-LPCs (Fig. 4D). These results indicated that inhibition of autophagy in LPCs promotes etoposide-induced senescence possibly through $\mathrm{p} 53 / \mathrm{p} 21$ signaling.

Overexpression of Beclin 1 in shBecn1-LPCs restored the functionality of stem cells

To fully verify that autophagy regulated the functionality of LPCs, we used myc-tagged full-length Beclin 1 plasmid to overexpress Beclin 1 in shBecn1-LPCs, as described in Materials and Methods. As shown in Fig. 5A, overexpression of Beclin 1 in shBecn1-LPCs increased the expression of LC3-II. Functional analysis demonstrated that overexpression of Beclin 1 in shBecn1-LPCs restored the hepatic differentiation capability, as indicated by PAS staining (Fig. 5B). In addition, overexpression of Beclin 1 rendered shBecn1-LPCs to be resistant to etoposide treatment, as indicated by decreased numbers of SA- $\beta$-gal ${ }^{+}$cells (Fig. 5C,D). Based on these results, we concluded that autophagy played a critical role in regulating the functionality of LPCs.

\section{Discussion}

In this study, we demonstrated that autophagy had a critical role in regulating LPC stemness and senescence. We found that inhibition of autophagy by knockdown of Atg5 or Becn1 impaired the clonogenic, proliferative and differentiation capability of LPCs, but had little impact on cell viability. In addition, inhibition of autophagy promoted the etoposideinduced senescence.

In this study, we inhibited autophagic activity in LPCs through knockdown of Atg5 or Becn1, which are essential elements required for autophagosome and autolysosome 


\section{Cellular Physiology Cell Physiol Biochem 2015;36:1163-1174 \begin{tabular}{ll|l} 
DOI: 10.1159/000430287 & $\begin{array}{l}\text { O 2015 S. Karger AG, Basel } \\
\text { www.karger.com/cpb }\end{array}$ \\
\hline
\end{tabular} \\ Cheng et al.: Autophagy Regulates Liver Progenitor Cells}

formation [11]. Either knockdown of Atg5 or Becn1 successfully downregulated the autophagic activity within LPCs, and negatively regulated the stemness of LPCs, suggesting that the effect of Atg5/Becn1-knockdown on LPC functionality was mediated through autophagy pathway, but not Atg5/Becn1-associated gene function.

In contrast to differentiated cells, where autophagy is usually induced as a consequence of stress, high autophagic activity is observed in adult skin and blood stem cells under physiological conditions [23]. And it is presumed that this constitutive autophagy is crucial for the maintenance of cellular homeostasis in various stem cells, including embryonic stem cells, hematopoietic stem cells, neural stem cells, cardiac stem cells, mesenchymal stem cells and cancer stem cells $[23,24]$. Inhibition of autophagy in those stem cells significantly impaired the self-renewal and differentiation capability [23]. In this study, we have also observed high level of autophagic activity in LPCs compared with that in hepatocytes. Inhibition of autophagic activity impaired the self-renewal and differentiation function, and promoted etoposide-induced senescence in LPCs. It is possible that the high autophagic activity prevents LPCs from damages during massive liver injury, when large amounts of hepatocytes are susceptible to such damages. Then, under such conditions, LPCs could activate and proliferate to support liver regeneration. Further studies including in vivo experiments and experiments using hepatic progenitor cells derived from mouse embryonic liver [25] are needed to fully prove the physiological role of autophagy in LPCs.

Cellular senescence is a state of cell cycle arrest induced by stress. Meanwhile, autophagy could also be induced by stress. Recent studies have demonstrated the interaction between autophagy and cellular senescence in somatic cells. It was reported that depletion of Atg7, Atg12, or lysosomal-associated membrane protein 2 by siRNA or shRNA resulted in premature senescence in primary human fibroblasts [26]. In addition, simultaneous inhibition of Cyclindependent kinase 4/6 and autophagy in human mammary epithelial cells enhanced the senescence response [27]. Here, we showed that impairment of autophagy led to senescence associated SA- $\beta$-gal activity in LPCs. In addition, the senescent phenotype induced by etoposide in shAtg5/shBecn1-LPCs was accompanied by the up-regulation of p53 and p21 mRNA expression, which was consistent with previous studies that reported etoposide induced cellular senescence in tumor cells through inducing p53 and p21 pathway $[28,29]$. Only those tumor cells with wild-type p53 exhibited senescent phenotype upon etoposide treatment or DNA damage $[28,29]$. Our results indicated the close interaction between autophagy and stress-induced cellular senescence within stem cells. However, during aging, whether autophagy plays a role in the regulation of stem cells remains unknown. Although it is known that autophagy activation extends lifespan in Drosophila melanogaster and mice [30-32], the exact function of autophagy within somatic cells and stem cells during aging still needs to be explored.

In conclusion, our findings indicate that autophagy plays a critical role in regulating LPC stemness. Further studies are needed to fully decipher the mechanisms by which autophagy regulates LPC functionality under physiological and pathological conditions.

\section{Acknowledgements}

This work was supported by Strategic Priority Research Program of the Chinese Academy of Sciences (XDA01040000), the Ministry of Science and Technology of China (2011CB966200, 2014CB943300), National Natural Science Foundation of China (81130057, 81071748, 81373164, 81190133, 31300708), the Program of Science and Technology Commission of Shanghai Municipality (12ZR1415900, 13ZR1423200, 14ZR1446300).

\section{Disclosure Statement}

The authors declare no competing financial interests.

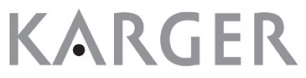




\section{Cellular Physiology Cell Physiol Biochem 2015;36:1163-1174 \begin{tabular}{l|l|l}
\hline DOI: 10.1159/000430287 & (C) 2015 S. Karger AG, Basel
\end{tabular} www.karger.com/cpb \\ Cheng et al.: Autophagy Regulates Liver Progenitor Cells}

\section{Reference}

1 Cai J, Weiss ML, Rao MS: In search of “stemness”. Exp Hematol 2004;32:585-598.

2 Feng D, Kong X, Weng H, Park O, Wang H, Dooley S, Gershwin ME, Gao B: Interleukin-22 promotes proliferation of liver stem/progenitor cells in mice and patients with chronic hepatitis B virus infection. Gastroenterology 2012;143:188-198.

3 Fausto N, Campbell JS: The role of hepatocytes and oval cells in liver regeneration and repopulation. Mech Dev 2003;120:117-130.

4 Akhurst B, Croager EJ, Farley-Roche CA, Ong JK, Dumble ML, Knight B, Yeoh GC: A modified cholinedeficient, ethionine-supplemented diet protocol effectively induces oval cells in mouse liver. Hepatology 2001;34:519-522.

5 Roskams T, Yang SQ Koteish A, Durnez A, DeVos R, Huang X, Achten R, Verslype C, Diehl AM: Oxidative stress and oval cell accumulation in mice and humans with alcoholic and nonalcoholic fatty liver disease. Am J Pathol 2003;163:1301-1311.

6 Lowes KN, Croager EJ, Abraham LJ, Olynyk JK, Yeoh GC: Upregulation of lymphotoxin beta expression in liver progenitor (oval) cells in chronic hepatitis C. Gut 2003;52:1327-1332.

7 Wang X, Lopategi A, Ge X, Lu Y, Kitamura N, Urtasun R, Leung TM, Fiel MI, Nieto N: Osteopontin induces ductular reaction contributing to liver fibrosis. Gut 2014;0:1-14.

$8 \quad$ Grompe M, Laconi E, Shafritz DA: Principles of therapeutic liver repopulation. Semin Liver Dis 1999;19:714.

9 Klionsky DJ, Emr SD: Autophagy as a regulated pathway of cellular degradation. Science (New York, N.Y.) 2000;290:1717-1721.

10 Mizushima N, Yoshimori T, Levine B: Methods in mammalian autophagy research. Cell 2010;140:313-326.

11 Boya P, Reggiori F, Codogno P: Emerging regulation and functions of autophagy. Nat Cell Biol 2013;15:713720.

12 Dang S, Xu H, Xu C, Cai W, Li Q, Cheng Y, Jin M, Wang RX, Peng Y, Zhang Y, Wu C, He X, Wan B, Zhang Y: Autophagy regulates the therapeutic potential of mesenchymal stem cells in experimental autoimmune encephalomyelitis. Autophagy 2014;10:1301-1315.

13 Hou J, Han ZP, Jing YY, Yang X, Zhang SS, Sun K, Hao C, Meng Y, Yu FH, Liu XQ, Shi YF, Wu MC, Zhang L, Wei LX: Autophagy prevents irradiation injury and maintains stemness through decreasing ROS generation in mesenchymal stem cells. Cell Death Dis 2013;4:e844.

14 Mortensen M, Soilleux EJ, Djordjevic G, Tripp R, Lutteropp M, Sadighi-Akha E, Stranks AJ, Glanville J, Knight S, Jacobsen SE, Kranc KR, Simon AK: The autophagy protein Atg7 is essential for hematopoietic stem cell maintenance. J Exp Med 2011;208:455-467.

15 Wang MJ, Chen F, Li JX, Liu CC, Zhang HB, Xia Y, Yu B, You P, Xiang D, Lu L, Yao H, Borjigin U, Yang GS, Wangensteen KJ, He ZY, Wang X, Hu YP: Reversal of hepatocyte senescence after continuous in vivo cell proliferation. Hepatology 2014;60:349-361.

16 Li WL, Su J, Yao YC, Tao XR, Yan YB, Yu HY, Wang XM, Li JX, Yang YJ, Lau JT, Hu YP: Isolation and characterization of bipotent liver progenitor cells from adult mouse. Stem Cells 2006;24:322-332.

17 Shin S, Walton G, Aoki R, Brondell K, Schug J, Fox A, Smirnova O, Dorrell C, Erker L, Chu AS, Wells RG, Grompe M, Greenbaum LE, Kaestner KH: Foxl1-Cre-marked adult hepatic progenitors have clonogenic and bilineage differentiation potential. Gene Dev 2011;25:1185-1192.

18 Zhao F, Zhang Y, Wang H, Jin M, He S, Shi Y, Guo Y, Zhang Y: Blockade of osteopontin reduces alloreactive $\mathrm{CD}^{+} \mathrm{T}$ cell-mediated graft-versus-host disease. Blood 2011;117:1723-1733.

19 Dimri GP, Lee X, Basile G, Acosta M, Scott G, Roskelley C, Medrano EE, Linskens M, Rubelj I, Pereira-Smith O, Peacocke M, Campisi J: A Biomarker That Identifies Senescent Human-Cells in Culture and in Aging Skin in-Vivo. P Natl Acad Sci USA 1995;92:9363-9367.

20 Xu C, Liu J, Hsu LC, Luo Y, Xiang R, Chuang TH: Functional interaction of heat shock protein 90 and Beclin 1 modulates Toll-like receptor-mediated autophagy. FASEB J 2011;25:2700-2710.

21 Leontieva OV, Gudkov AV, Blagosklonny MV: Weak p53 permits senescence during cell cycle arrest. Cell cycle 2010;9:4323-4327.

22 Deng Y, Chan SS, Chang S: Telomere dysfunction and tumour suppression: the senescence connection. Nat Rev Cancer 2008;8:450-458. 


\section{Cellular Physiology Cell Physiol Biochem 2015;36:1163-1174 and Biochemistry

23 Salemi S, Yousefi S, Constantinescu MA, Fey MF, Simon HU: Autophagy is required for self-renewal and differentiation of adult human stem cells. Cell Res 2012;22:432-435.

24 Guan JL, Simon AK, Prescott M, Menendez JA, Liu F, Wang F, Wang C, Wolvetang E, Vazquez-Martin A, Zhang J: Autophagy in stem cells. Autophagy 2013;9:830-849.

25 Bi Y, He Y, Huang J, Su Y, Zhu GH, Wang Y, Qiao M, Zhang BQ, Zhang H, Wang Z, Liu W, Cui J, Kang Q, Zhang Z, Deng Y, Li R, Zhang Q, Yang K, Luu HH, Haydon RC, He TC, Tang N. Functional characteristics of reversibly immortalized hepatic progenitor cells derived from mouse embryonic liver. Cell Physiol Biochem 2014;34(4):1318-1338.

26 Kang HT, Lee KB, Kim SY, Choi HR, Park SC: Autophagy impairment induces premature senescence in primary human fibroblasts. PloS One 2011;6:e23367.

27 Brown NE, Jeselsohn R, Bihani T, Hu MG, Foltopoulou P, Kuperwasser C, Hinds PW: Cyclin D1 activity regulates autophagy and senescence in the mammary epithelium. Cancer Res 2012;72:6477-6489.

28 Marusyk A, Wheeler LJ, Mathews CK, DeGregori J: p53 mediates senescence-like arrest induced by chronic replicational stress. Mol Cell Biol 2007;27:5336-5351.

29 te Poele RH, Okorokov AL, Jardine L, Cummings J, Joel SP: DNA damage is able to induce senescence in tumor cells in vitro and in vivo. Cancer Res 2002;62:1876-1883.

30 Pyo JO, Yoo SM, Ahn HH, Nah J, Hong SH, Kam TI, Jung S, Jung YK: Overexpression of Atg5 in mice activates autophagy and extends lifespan. Nat Commun 2013;4:2300.

31 Kapahi P, Zid BM, Harper T, Koslover D, Sapin V, Benzer S: Regulation of lifespan in Drosophila by modulation of genes in the TOR signaling pathway. Curr Biol 2004;14:885-890.

32 Bjedov I, Toivonen JM, Kerr F, Slack C, Jacobson J, Foley A, Partridge L: Mechanisms of life span extension by rapamycin in the fruit fly Drosophila melanogaster. Cell Metab 2010;11:35-46. 